\title{
The Study and Simulation on Effects of UHV access to Power Grid
}

\author{
Mengyao Zhang ${ }^{1, a}$, Xin Wang ${ }^{2, b^{*}}$, Min $\mathrm{Li}^{3}$ \\ ${ }^{1}$ Logistics Engineering College, Shanghai Maritime University, Shanghai, China \\ ${ }^{2}$ Center of Electrical \& Electronic Technology, Shanghai Jiao Tong University, Shanghai, China \\ ${ }^{3}$ Guang'an Power Supply Company of State Grid Sichuan Electric Power Company, China \\ a529769621@qq.com, bwangxin26@sjtu.edu.cn \\ *corresponding author: wangxin26@sjtu.edu.cn
}

Keywords: Ultra-High Voltage; Stability Analysis; Voltage Adjustment; Sensitivity Analysis

Abstract. The access of Ultra-High Voltage (UHV) line must have a great impact on security and stability of power grid. In this paper, to access a 1000kV UHAC line to a power grid, the stability and security of power grid are analyzed and a sensitivity method is proposed to solve the problem of threshold-crossing voltage. Firstly, it establishes the model of a power grid by using PSD-BPA software and makes analysis of power flow and N-1 static security of lines of 500kV and above. Secondly, by setting up three-phase short circuit malfunctions near the impact point of UHV grid and using the stable curve analysis tools, transient stability of power grid is analyzed. Thirdly, based on the analysis results of the security and stability above, the $220 \mathrm{kV}$ grid arises the problem of threshold-crossing voltage. Combined with the sensitivity analysis, a node voltage adjustment program is proposed to solve this problem. It calculates sensitivity value of the reactive power compensation for the threshold-crossing voltage nodes nearby and determines the amount of reactive power compensation. Combined with the methods of reactive power compensation for load nodes and transformer ratio adjustment, it adjusts the level of voltage to more reasonable threshold. Finally, the simulation on the PSD-BPA software proves that the program can effectively adjust the level of voltage to more reasonable range.

\section{Introduction}

UHV has many advantages, such as, high transmission efficiency, low loss, small corridor area, improvement of the stability of the receiving system. UHV power grid has a high margin of safety and stability [1]. With the rapid development of UHV power grid, more UHV lines will access to power grids. The access of UHV line can effectively fulfill the electricity provision to the receiving power grid, but also changes the network structure of the power grid. It has great influence on the security and stability of the power grid. Due to the excessive electricity outside, the receiving power grid will obviously have problems with the voltage stability. Therefore, it is necessary to adjust and control the threshold-crossing voltage.

In [2], the bus voltage control near UHV area was in accordance with the minimum principles of reactive power exchange to develop reactive power compensation switching strategy, but under the control of the policy above, control range was small and the number of device operation was limited. As the strategy proposed in [3], although it could achieve optimal partial local control by reactive power compensation, but it couldn't meet the real-time balancing requirements. In [4], if the voltage level of Nanyang UHV grid and Central China 500kV power grid could be simultaneously controlled, it was need to reduce operating standards of Nanyang UHV gird. However, these methods above have defects. So [5] introduced a new sensitivity analysis method to sort, according to the severity of voltage crashes. In [6], the comprehensive sensitivity analysis of load voltage nodes and short circuit current was used to sort, to select the larger sensitivity value to take the voltage control. In [7] it showed that the use of sensitivity analysis in distributed grid could control bus voltage into the range.

In this paper, to access a 1000kV UHAC line to a power grid, the stability and security of power grid are analyzed and a sensitivity method is proposed to solve the problem of threshold-crossing 
voltage. Firstly, it establishes the power grid model by using PSD-BPA software and makes analysis of static security and transient stability. Secondly, based on the analysis results above, it arises the problem of threshold-crossing voltage. Combining the sensitivity analysis method with the methods of reactive power compensation and transformer ratio adjustment, the node voltage adjustment program is proposed to solve this problem. Finally, the simulation on the PSD-BPA software proves that the program can be effectively feasible.

\section{Research foundation}

Grid Computing. In this paper, the model for $1000 \mathrm{kV}$-UHAC line access to power grid is built on the PSD-BPA simulation software. $1000 \mathrm{kV}$ and $500 \mathrm{kV}$ grids take close-loop operation mode and constitute the three electromagnetic loop network with $220 \mathrm{kV}$ grid.

Calculation Model. The generator model of power grid is the changing model of cross-axis sub-transient potential $E q^{\prime \prime}$ with damping winding and direct-axis potential $E d "$, which considers the effect of excitation, PSS and speed control system. The load model is $60 \%$ constant power and $40 \%$ constant impedance load model. Active, reactive frequency factor is 1.8、2.0.

\section{Security and Stability Analysis}

Results and Analysis of Power Flow Calculation. Use the PSD-BPA program to analyze and calculate the power flow of power grid. The picture of power flow distribution is shown in Fig. 1.

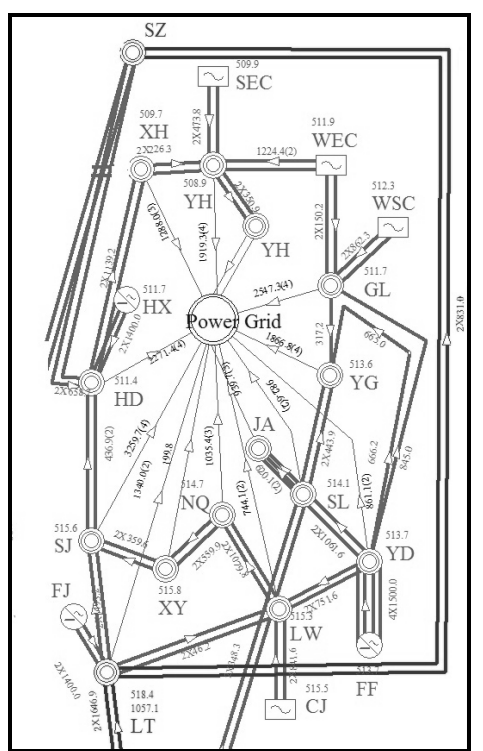

Fig.1. Power flow of UHV access to power grid

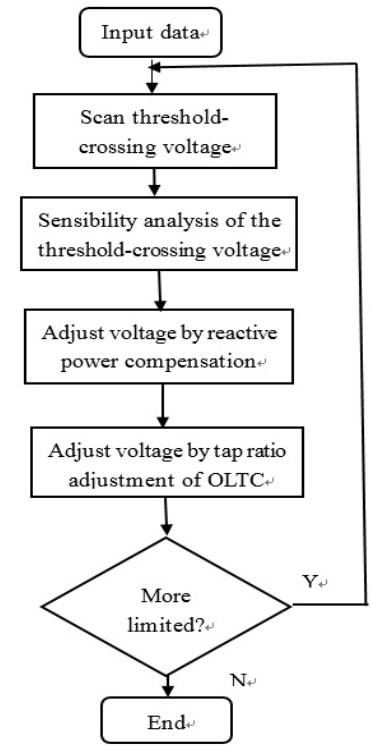

Fig.2. Voltage adjustment flowchart

Based on the results of the analysis, lines of $500 \mathrm{kV}$ and above near the impact point of UHV grid and main transformers do not exist overload or heavy load problem and $500 \mathrm{kV}$ grids do not exist threshold-crossing voltage problem. However, $220 \mathrm{kV}$ lines connected to LT node overload.

N-1 Static Security Analysis. Use the PSD-BPA software to scan N-1 malfunctions of 500kV and $1000 \mathrm{kV}$ lines of UHV access to power grid. After the scan, results show that when a loop is broken, $500 \mathrm{kV}$ and $1000 \mathrm{kV}$ lines of the receiving power grid will not exist conditions of overload.

Transient Stability Analysis. In this paper, the method of transient stability analysis is utilized to calculate the versus time curve of voltage and the relative angle among the generator rotors after the disturbance [8]. Considering the most serious malfunction, it sets up three-phase short circuit malfunctions for one or two loops on UHV 1000kV line (SZ-LT) and two 500kV lines (SZ-HD and SJ-LT) near the impact point of UHV line to analyze transient stability of power grid. The malfunction duration is $0.1 \mathrm{~s}$ [9]. The simulation results show that after three-phase short circuit malfunctions occur 
for one or two loops, UHV line and $500 \mathrm{kV}$ lines near the impact point of UHV line can maintain transiently stable.

\section{Voltage adjustment programs}

Applications of Sensitivity Analysis on the Voltage Adjustment. Sensitivity analysis is to use differential relations of certain physical quantities in system, to obtain sensitivity of the dependent variable on the independent variable [10]. It is widely used in voltage stability studies. The sensitivity indicators of determining voltage stability of power system are $\frac{d U_{D}}{d Q_{G}}, \frac{d U_{D}}{d Q_{D}}$, etc.[11]

(1) Sensitivity matrix of the generator reactive power $\Delta Q_{G}$ to the load node voltage $\Delta U_{D}$

$$
\left[\begin{array}{l}
\Delta U_{D} \\
\Delta U_{G}
\end{array}\right]=-\left[\begin{array}{ll}
L_{D D} & L_{D G} \\
L_{G D} & L_{G G}
\end{array}\right]^{-1}\left[\begin{array}{c}
\Delta Q_{D} \\
\Delta Q_{G}
\end{array}\right],\left[\begin{array}{l}
R_{D D} R_{D G} \\
R_{G D} R_{G G}
\end{array}\right]=-\left[\begin{array}{cc}
L_{D D} L_{D G} \\
L_{G D} L_{G G}
\end{array}\right]^{-1}
$$

In Eq.1, when the power output of generator reactive changes, assume active power load nodes constant, which is $\Delta Q_{D}=0$. Then, $\left[\begin{array}{c}\Delta U_{D} \\ \Delta U_{G}\end{array}\right]=\left[\begin{array}{c}R_{D G} \\ R_{G G}\end{array}\right] \Delta Q_{G}$, which means $\Delta U_{D}=R_{D G} \Delta Q_{G}$.

(2) Sensitivity matrix of reactive power compensation $\Delta Q_{D}$ to load node voltage $\Delta U_{D}$

When there is increasing reactive power compensation for the load node, assume the output of generator reactive power unchanged, which is $\Delta Q_{G}=0$. Then, $\Delta U_{D}=R_{D D} \Delta Q_{D}$.

(3) Sensitivity matrix of tap ratio adjustment $\Delta t$ of on load tap changing transformer (OLTC) to load node voltage $\Delta U_{D}$

Assuming that transformer ratio has changed $\Delta t$, if the generator bus voltage is constant, injection of load node reactive power is also unchanged, the load node voltage will change. Since the relationship of sensitivity is Eq.2, then $\Delta U_{D}=T_{D t} \Delta t$.

$$
\Delta Q_{D}=\left[\frac{\partial \Delta Q_{D}}{\partial U_{D}^{T}}\right] \Delta U_{D}+\left[\frac{\partial \Delta Q_{D}}{\partial t^{T}}\right] \Delta t=0
$$

Voltage Adjustment Process. Voltage adjustment process is shown in Fig.2.

Voltage Violations Example Analysis. Set 0.95 for lower limit voltage and 1.05 for upper limit voltage. All the actual voltage of $220 \mathrm{kV}$ grids has arisen more limited phenomenon. In the paper, pick out five central load nodes for analysis. The sensitivity of load nodes are shown in Table1.

Table 1. Sensitivity analysis of load nodes

\begin{tabular}{|c|c|c|c|c|c|c|}
\hline $\begin{array}{c}\text { Node } \\
\text { name }\end{array}$ & $\begin{array}{c}\text { Reactive power } \\
\text { compensation[MVA] }\end{array}$ & $\begin{array}{c}\text { Sensitivity } \\
\text { values }\end{array}$ & \multicolumn{2}{c|}{$\begin{array}{c}\text { Voltage before } \\
\text { adjustment[pu/kV] }\end{array}$} & \multicolumn{2}{c|}{$\begin{array}{c}\text { Voltage after } \\
\text { adjustment[pu/kV] }\end{array}$} \\
\hline NQ_4 & -60 & 0.0161 & 1.087 & 39.11 & 1.057 & 37.98 \\
\hline XY_1 & -60 & 0.0172 & 1.153 & 41.5 & 1.12 & 40.13 \\
\hline YG_3 & -60 & 0.0160 & 1.084 & 39.01 & 1.055 & 37.88 \\
\hline SJ_1 & -60 & 0.0157 & 1.125 & 40.51 & 1.096 & 39.32 \\
\hline YH_2 & 135 & 0.0179 & 0.955 & 34.38 & 1.019 & 36.58 \\
\hline
\end{tabular}

Comparison of the sensitivity values, select the maximum sensitivity value of the load node for reactive power compensation. After the reactive power compensation shown above, there are still some threshold-crossing voltage nodes left. Adjust tap ratio of main OLTC near these nodes until these threshold-crossing voltage nodes reach within a reasonable range. There is no data of the threshold-crossing voltage shown in the PSD-BPA software, which means the power grid does not exist the load node of the threshold-crossing voltage. 


\section{Conclusions}

Based on the model of UHV access to power grid, the power flow calculation shows that $500 \mathrm{kV}$ and $1000 \mathrm{kV}$ lines do not exist overload problems, but $220 \mathrm{kV}$ grids still have overload problems. N-1 check shows that $500 \mathrm{kV}$ and $1000 \mathrm{kV}$ lines of power grid will not have any overload problem. The transient stability analysis show that after three-phase short circuit malfunctions of the three lines near the impact point of UHV grid, the power system can maintain transient stability. The use of sensitivity value of reactive power of load nodes and combination reactive power compensation with transformer tap adjustment can adjust these threshold-crossing voltage to more reasonable range.

\section{Acknowledgements}

This work is supported by the Key Project of NSFC (No. 61533012), the Shanghai Natural Science Foundation (14ZR1421800), the State Key Laboratory of Synthetical Automation for Process Industries.

\section{References}

[1] Yinbiao Shu, Wenliang Zhang, Xiaoxin Zhou, Security Evaluation of UHV Synchronized Power Grid, Proceedings of the CSEE. 27(2007)1-6. In Chinese.

[2] Xiaojun Tang, Wenchao Zhang, Liping Qiu, Influence of Nanyang Substation to Ultra high Voltage and Henan Power Grid, Proceedings of the CUS-EPSA. 21(2009)62-68. In Chinese.

[3] Xiaojun Tang, Huiyuan Chen, Shuling Yao, Reactive Power and Voltage Control Strategy for UHVAC Power Grid Based on Improved Economic Voltage Difference, Power System Technology. 37(2013)673-677. In Chinese.

[4] Xiaojie Pan, Dichen Liu, Changqing Lin, Study of voltage adjust in Central China Grid to suit for huge power transmitted southward through UHV project, Power System Protection and Control. 37(2009)23-26. In Chinese.

[5] Alexander. J. Flueck, A New Power Sensitivity Method of Ranking Branch Outage Contingencies for Voltage Collapse, IEEE Transactions on Power System. 17(2002)265-270.

[6] Fei Dai, Ting Cui, Jian Xu, Based on a comprehensive analysis of voltage sensitivity correction control, Electric Power Automation Equipment. 31(2011)15-20. In Chinese.

[7] V. Calderaro, V. Galdi, Distributed Generation and local voltage regulation: an approach based on sensitivity analysis, IEEE PES Innovative Smart Grid Technologies Conference Europe. 2011.

[8] V. N. Avramenko, Power System Stability Assessment for Current States of the System, IEEE Russia Power Tech, Power Tech. 2005.

[9] Yao Shao, Yong Tang, Xiaohong Guo, Transient Voltage Stability Analysis of East China Receiving-End Power Grid With Multi-Infeed HVDC Transmission Lines, Power System Technology. 35(2011)50-55. In Chinese.

[10] Fengxian Miao, Zhizhong Guo, A survey of sensitivity technique and its application in power systems analysis and control, Relay. 35(2007)72-76. In Chinese.

[11] R.A. Schlueter, M.W. Chang, Methods for determining proximity to Voltage collapse, Power Systems, IEEE Transactions on Power System. 1(1991)285-292. 